\title{
ANLN promotes cell migration and invasion through RhoA-ROCK signaling in cervical cancer
}

\author{
Congzhe Hou, Zhen Liang, Yongxia Yang, Yunhai Yu, Tingting Liang, Lin Zhu, Ping Zhang
}

Department of Gynecology, The Second Hospital, Cheeloo College of Medicine, Shandong University, Jinan, Shandong, China

Submitted: 28 August 2020

Accepted: 25 March 2021

Arch Med Sci

DOI: https://doi.org/10.5114/aoms/134899

Copyright $\odot 2021$ Termedia \& Banach

\section{Abstract}

Introduction: Anillin actin binding protein (ANLN) is involved in various human cancers. It is often upregulated in various cancers, including cervical cancer (CC). However, the exact role of ANLN in the modulation of CC and the underlying molecular mechanism remain unknown. In this study, we aimed to investigate the effects of ANLN on the proliferation, migration, and invasion of CC cells, as well as to determine the molecular mechanisms underlying these effects.

Material and methods: ANLN expression levels were analyzed in normal cervical and CC specimens using public databases and tissue samples. The prognosis was determined using the TCGA database. Cell proliferation, migration and invasion were measured by Edu assay, wound-healing assay and transwell assay, respectively. Immunofluorescence was used to examine the influence of ANLN inhibition on actin stress fiber integrity. Western blots were used to measure the protein expression.

Results: ANLN expression levels in CC were higher than those in normal tissues, and ANLN overexpression was highly correlated with poor prognosis. ANLN knockdown inhibited CC cell proliferation, migration, and invasion in vitro, while ANLN overexpression led to an inverse biological phenotype. Immunofluorescence showed that ANLN inhibition could influence actin stress fiber integrity. ANLN expression was positively correlated with ROCK1 and ROCK2 expression in CC. Overexpression of ANLN activated RhoA and upregulated ROCK1 and ROCK2. Furthermore, ROCK1 and ROCK2 expression levels were also impeded by $\mathrm{Y} 27632$, which is a specific inhibitor of RhoA. They also weakened the migration and invasion ability in ANLN-overexpression HeLa cells.

Conclusions: ANLN promotes cell migration and invasion through RhoAROCK signaling in CC.

Key words: cervical cancer, anillin actin binding protein, RhoA.

\section{Introduction}

Cervical cancer (CC) is one of the most common gynecological malignancies in the world. Despite the implementation of modified cervical cancer screening programs [1], approximately 570000 cases of CC and 311000 CC-related deaths still occurred in 2018 [2]. About $85 \%$ of new cases occur in developing countries and account for the second highest incidence of female malignancies [3]. The current major therapies for CC, including surgery, chemotherapy, radiotherapy, and biological targeted therapy, which have improved the survival rate of patients with CC, still

\author{
Corresponding author: \\ Lin Zhu, \\ Ping Zhang \\ Department \\ of Gynecology \\ The Second Hospital \\ Cheeloo College \\ of Medicine \\ Shandong University \\ Jinan, Shandong 250033 \\ E-mail: 121356189@qq.com, \\ sdey_zhulin2015@163.com
}


have a limited curative effect for some patients [4]. Local tumor recurrence and distant metastasis are still the main causes of death among patients.

One of the first steps in the spread of metastatic cancers is the movement of cancer cells into the vasculature and tissues surrounding the tumor. Cytoskeleton plays an essential role in the migration and spread of tumor cells [5]. Novel therapeutic strategies can be provided from a deep understanding of the mechanisms of cytoskeletal regulation to block the metastatic progression and improve the survival rates of patients. Anillin actin-binding protein (ANLN), an actin binding protein [6], plays a vital role in cellular processes such as cell proliferation, movement, and cytokinesis [7]. It frequently contributes to the development of cancer. For example, ANLN can regulate the proliferation of gastric cancer cells, and it is identified to be a Wnt/-catenin responsive gene in gastric cancer [8]. In bladder urothelial carcinoma, ANLN downregulation impairs cell proliferation and metastasis [9]. ANLN knockdown represses cell growth, and overexpression promotes cell motility in non-small cell lung cancer cells [10]. Additionally, ANLN overexpression promotes metastasis and progression in breast cancer [11], colorectal cancer [12] and pancreatic cancer [13]. Moreover, ANLN has been identified as a key candidate gene in CC as determined by integrated bioinformatic analysis [14]. However, the exact role of ANLN in the modulation of CC and the underlying molecular mechanism remain unknown. In this study, we aimed to investigate the effects of ANLN on the proliferation, migration, and invasion of CC cells, as well as to determine the molecular mechanisms underlying these effects.

\section{Material and methods}

\section{Public databases}

The series matrix files of GSE7410, GSE63514, and GSE67522 were downloaded from the public Gene Expression Omnibus (http://www.ncbi.nlm. nih.gov/geo/) database. The platforms that these series matrix files were based on were GPL1708 (Agilent-012391 Whole Human Genome Oligo Microarray G4112A), GPL570 (Affymetrix Human Genome U133 Plus 2.0 Array), and GPL10558 (Illumina Human HT-12 V4.0 expression beadchip). R software (version 3.4.0, https://www.r-project.org/) was used to process all GEO data, and differentially expressed genes between CC and noncancerous samples were identified by the limma package (http://www.bioconductor.org/packages/release/ bioc/html/limma.html). An adjusted $p<0.05$ and the absolute $\log _{2}$ fold change $|\log 2 \mathrm{FC}|>1$ were considered statistically significant.

RNA-sequencing data for tumor tissue were downloaded from the University of California,
Santa Cruz Xena browser (https://xenabrowser. net). The "Survival" package was loaded in RStudio, and Kaplan-Meier curves were employed on the follow-up data from The Cancer Genome Atlas (TCGA). The statistical significance of the difference in survival was assessed by the log-rank test between two groups. $P<0.05$ indicated a statistically significant difference.

\section{Collection of samples from patients with CC}

This study was approved by the medical ethics practices and the Human Ethics Review Board of the Second Hospital of Shandong University, China. All specimens were obtained between January 2018 and June 2019 from patients with pathologically confirmed CC after surgery at the Second Hospital of Shandong University. Informed consent was obtained from all study subjects before sample collection, and these samples were used according to ethical standards.

\section{Cell culture}

HeLa, Caski, SiHa, and normal cervical cells (CRL-2614) were obtained from the Cell Bank of the Chinese Academy of Sciences (Shanghai, China). These cells were grown at $37^{\circ} \mathrm{C}$ in $5 \% \mathrm{CO}_{2}$ using RPMI-1640 medium supplemented with $10 \%$ fetal bovine serum (FBS). Transfection was performed with Lipofectamine 2000 reagent (Invitrogen; Thermo Fisher Scientific, Waltham, MA, USA) following the protocols of the manufacturer.

\section{Reverse transcription quantitative polymerase chain reaction (qRT-PCR) analysis}

Total RNA was isolated from tissue and cells using TRIzol reagent (Invitrogen), and $1 \mu \mathrm{g}$ of RNA was reverse transcribed into cDNA using a PrimeScript RT reagent kit (Takara Biotechnology Co., Ltd., Dalian, China). qPCR was conducted using SYBRGreen dye mix (Invitrogen) in accordance with the instructions of the manufacturer. The ANLN sense and antisense primers, that is, 5'-GCTGCGTAGCTTACAGACTTAGC-3' and 5'-AAGGCGTTTAAAGGTGATA GGTG-3' [12], respectively, were used. The GAPDH sense and antisense primers were $5^{\prime}$-CTCAAGATCATCAGCAAT- 3 ' and 5'-CGATACCAAAGTTGTCAT- $3^{\prime}$, respectively. The relative expression level of ANLN was calculated using the $2^{-\Delta \Delta c t}$ method and normalized to the internal reference gene GAPDH.

\section{Wound-healing assays}

Cells were seeded in six-well plates and grown until $90 \%$ confluence was reached. The cell monolayers were wounded by scraping them with a $200 \mu \mathrm{l}$ pipette tip. The results were observed by 
a phase-contrast microscope either immediately or $72 \mathrm{~h}$ after wounding. All experiments were repeated three times.

\section{Invasion assay}

A Transwell chamber coated with $50 \mu \mathrm{l}$ of Matrigel was placed into a 24-well plate and incubated for $30 \mathrm{~min}$ at $37^{\circ} \mathrm{C}$. Subsequently, $1 \times 10^{5}$ cells were trypsinized, added to chambers, and cultured in serum-free medium. The bottom was filled with medium containing $10 \%$ FBS. The cells on the upper surface of the membrane were removed, and the migrated cells were fixed with $100 \%$ methanol for 30 min after $24 \mathrm{~h}$. The bottom cells were stained with hematoxylin for $20 \mathrm{~min}$. The cell images were captured under a phase-contrast microscope.

\section{Immunofluorescence assay}

CC cell slides transfected with siRNA-control and SiRNA-ANLN were fixed with $4 \%$ paraformaldehyde for $30 \mathrm{~min}$. They were permeated with $0.1 \%$ Triton-X100 for $10 \mathrm{~min}$. The slides were sealed with phalloidin at room temperature for $1 \mathrm{~h}$. The CC cell slides were then sealed with a drop sealant. Lastly, they were photographed under a confocal laser microscope. The experiment was repeated three times.

\section{Western blot}

Total protein of the cells was isolated with RIPA tissue lysis Buffer (Solarbio, Beijing, China) in accordance with the operating manual. The concentration of the obtained protein was measured with BCA. Equal amounts (50 mg) of protein were separated on a $10 \%$ sodium dodecyl sulfate polyacrylamide gel and transferred to polyvinylidene fluoride membranes (Thermo Scientific). Membranes were incubated with primary rabbit antibodies against ANLN antibody (1 : 3000, 66643-1; Proteintech), RhoA (1 : 1000, 2117; Cell Signaling Technology, Danvers, MA, USA), and $\beta$-actin (1 : 5000, AC026; ABclonal) at $37^{\circ} \mathrm{C}$ overnight. The membranes were incubated with horseradish peroxidase-conjugated goat anti-rabbit secondary antibodies ( 1 : 10000; ABclonal) for $1 \mathrm{~h}$ at room temperature. The signals were detected with an enhanced chemiluminescence detection reagent (Thermo Scientific). The bands were quantified via densitometry and analyzed with Quantity One software (Bio-Rad Laboratories, Hercules, CA, USA). Relative protein levels were normalized to $\beta$-actin.

\section{RhoA activation assay}

A RhoA Activation Assay Biochem Kit (Cytoskeleton, Inc., Denver, Co., USA) was used to detect
RhoA activity following the protocol provided by the reagent manufacturer.

\section{Statistical analysis}

Statistical analyses were calculated using SPSS version 20.0 (IBM SPSS Statistics). All measurement data were presented as the mean \pm SD of three individual experiments. Statistical analyses were performed using Student's $t$-test between the two groups; when appropriate, the Mann-Whitney $t$-test was used. $P<0.05$ was considered to indicate a statistically significant result. The Kaplan-Meier method was used to evaluate prognostic results, and statistical analysis was performed through the log-rank test. $\mathrm{R}$ version 3.5.1 was used for statistical analyses.

\section{Results}

\section{ANLN was upregulated in CC tissues and correlated with poor prognosis}

ANLN was significantly upregulated in CC compared with the corresponding normal tissue by analyzing three datasets, namely, GSE7410 (Figure 1 A), GSE63514 (Figure 1 B), and GSE67522 (Figure 1 C). Consistently, qRT-PCR analysis demonstrated that ANLN was significantly overexpressed in the CC tissue (Figure 1 D) obtained from our hospital and cell lines (Figure 1 E) compared with the normal cervical tissue and cell line. Furthermore, TCGA database analysis of ANLN transcription by GEPIA (http://gepia.cancer-pku.cn/detail.php) also supported these results, and no statistically significant differences were found in different histological subtypes of CC (Figure 2). These results suggested that ANLN might be an oncogene in CC progression. We used the Kaplan-Meier and logrank tests to investigate the relationship between ANLN expression level and the overall survival (OS) of patients (Figure $1 \mathrm{~F}$ ). The TCGA datasets showed that patients with low ANLN had long OS.

\section{ANLN regulated CC cell proliferation, migration, and invasion}

To analyze the effects of ANLN on the malignant phenotypes of the CC cells, we performed loss function assays. Western blot analysis showed that si-ANLN transfection significantly suppressed ANLN expression in HeLa and Caski cells (Figures $3 \mathrm{~A}, \mathrm{~B})$. The effects of low ANLN expression on the proliferation of the CC cell lines (e.g., CaSki and HeLa) were investigated. The results of an Edu assay revealed that transfection with si-ANLN reduced the proliferation of the CC cells (Figures $3 \mathrm{C}$, D). Thus, ANLN promoted CC proliferation.

Migration and invasion assays were carried out to investigate the effects of ANLN on CC 
A

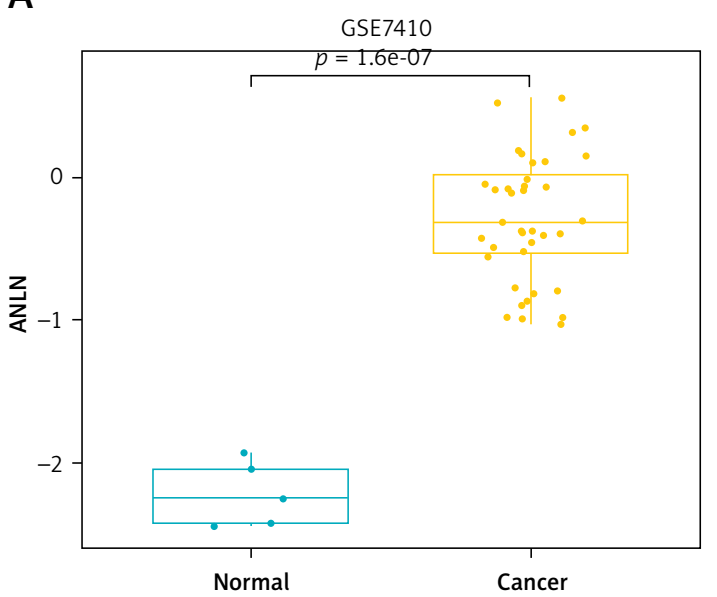

C

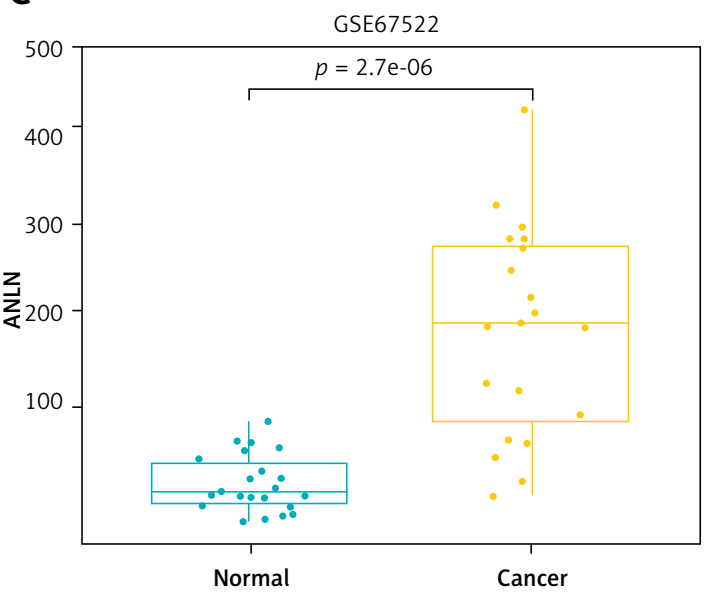

$\mathrm{E}$

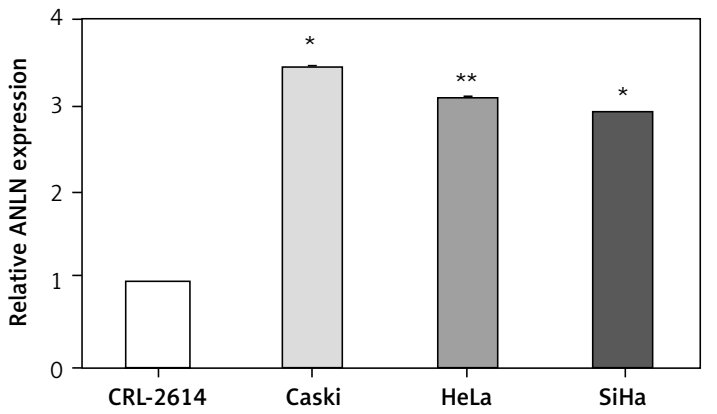

B

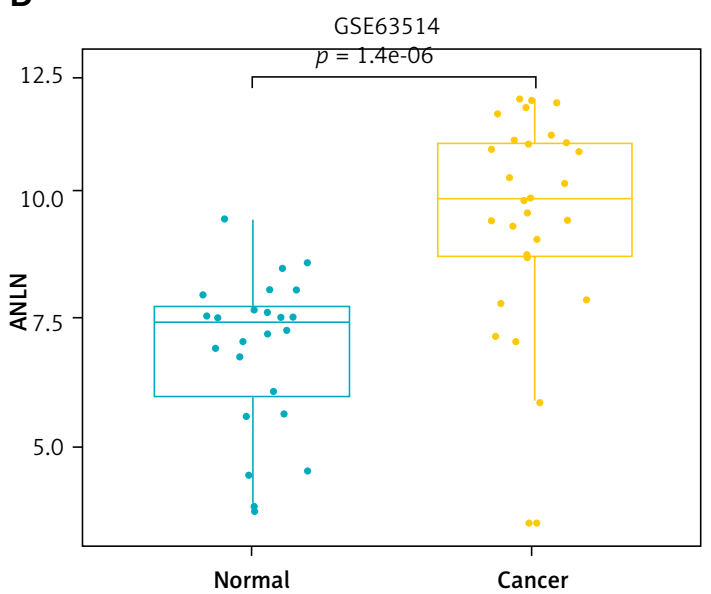

D

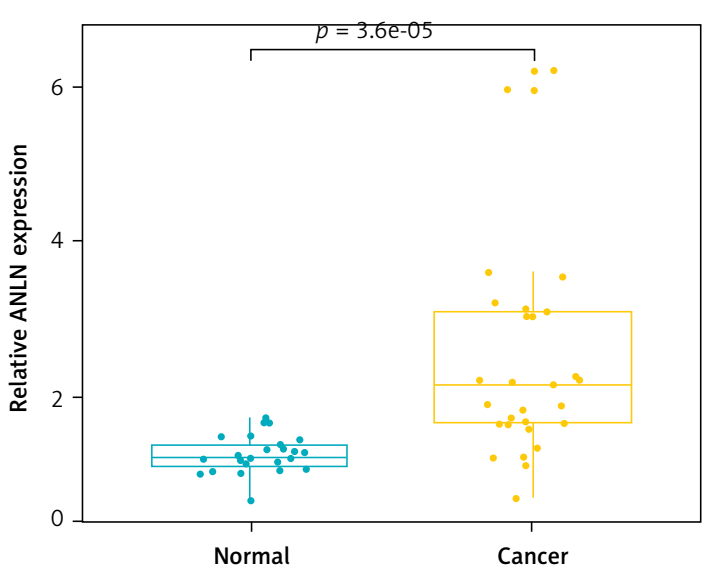

$\mathrm{F}$

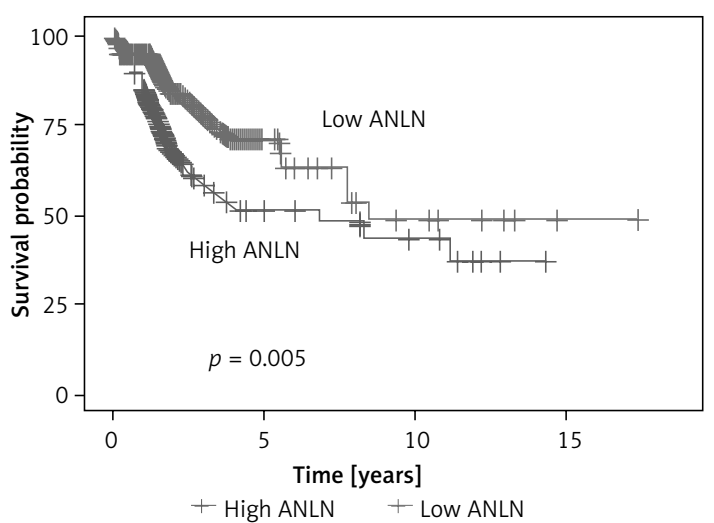

Figure 1. ANLN expression in CC tissue and cell lines. A-C - Box plots exhibited increased ANLN expression levels in CC compared with normal samples obtained from three microarray datasets $\left(\mathbf{A}-p=1.6 \times 10^{-7}\right.$ for GSE7410, B $-p=1.4 \times 10^{-6}$ for GSE63514, C $-p=2.7 \times 10^{-6}$ for GSE67522). D - qRT-PCR analysis of ANLN expression in CC and normal tissues. Data were reported as means $\pm \mathrm{SE}(N$ [normal] $=26, N$ [cancer] $=29), p=3.6 \times 10^{-5}$. E - ANLN expression in cervical cancer cell lines HeLa, Caski, SiHa, and CRL-2614. ${ }^{*} p<0.05 ;{ }^{* *} p<0.01$. F - Kaplan-Meier survival curves for OS time difference in patients with CC from the TCGA datasets $(p=0.005)$

cells. CC cells in the si-ANLN group exhibited decreased migratory (Figures $3 \mathrm{E}, \mathrm{F}$ ) and invasive abilities (Figures $3 \mathrm{G}, \mathrm{H}$ ) compared with control cells. These effects were statistically significant in each cell type.
To further verify these effects, we also constructed an ANLN-overexpression lentivirus and infected Hela cells. Consistent with the results from the siRNA experiments, ANLN overexpression had stronger proliferation, migration and 
A

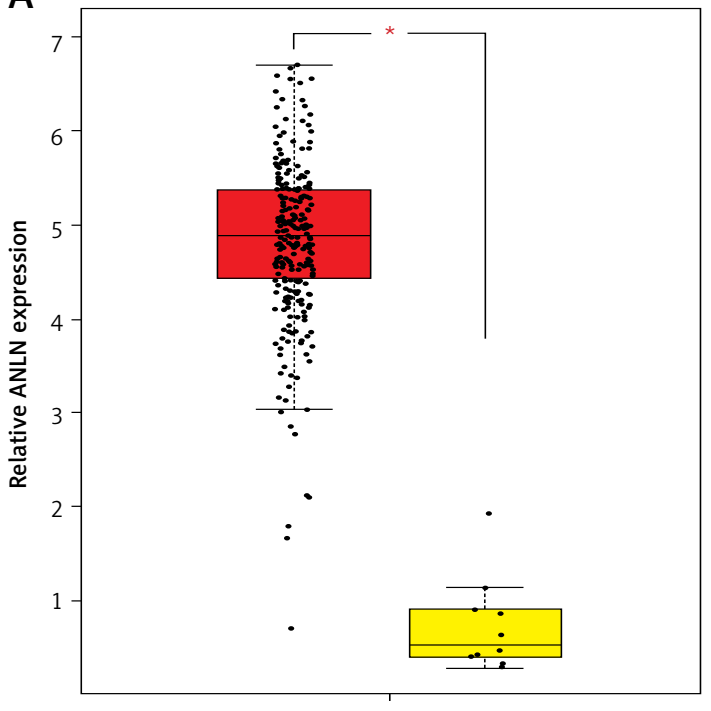

$\operatorname{CESC}(\operatorname{num}(\mathrm{T})=306 ; \operatorname{num}(\mathrm{N})=13)$
B

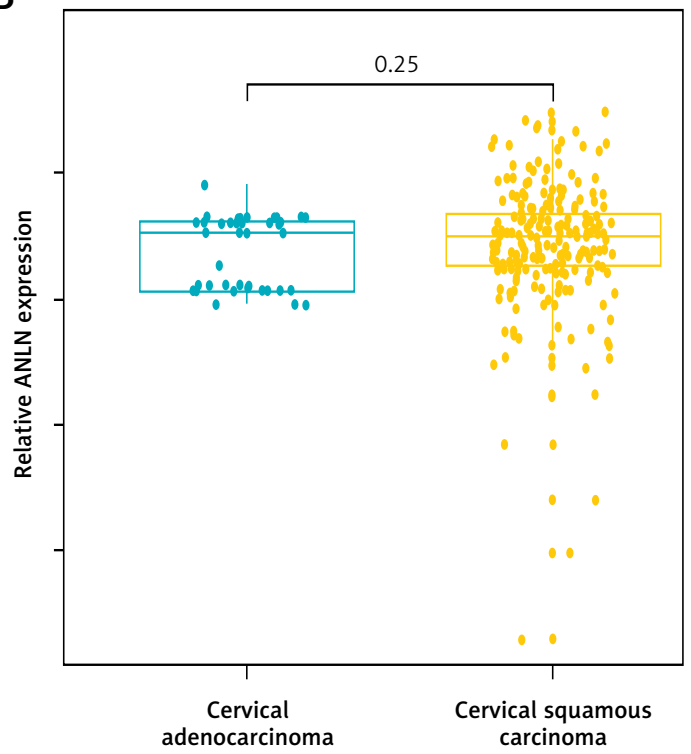

Figure 2. A - Expression of ANLN in CC and normal cervical tissues was analyzed using the GEPIA online database $\left({ }^{*} p<0.05\right)$. B - The level of ANLN expression in different histological types of cervical cancer from the TCGA database was analyzed $(p>0.05)$

invasion capabilities in HeLa cells than in control HeLa cells (Figures $3 \mathrm{I}-\mathrm{K}$ ).

\section{Actin stress fiber integrity is disrupted by ANLN knockdown}

F-actin is extremely important to maintain the normal morphology of cells. It is also closely correlated with tumor invasion and metastasis. We investigated whether ANLN can regulate actin cytoskeleton by examining F-actin staining in CC cells with ANLN knockdown (Figure 4). Immunofluorescence results showed that a partial loss of stress fiber integrity was evident in si-ANLN cells compared with control cells. These results indicated that the decreased ANLN expression levels influenced the stress fiber formation, which could affect cell invasion and migration.

\section{ANLN regulated $C C$ migration and invasion potential via the RhoA-ROCK pathway}

To further reveal the molecular mechanisms by which ANLN regulates cell invasion and migration, we applied bioinformatics analysis. RhoA-ROCK is one of the most essential mechanisms for migration and invasion. The relationship between ANLN and RhoA effectors ROCK 1 and ROCK 2 in patients with CC were analyzed by using the online web tool GEPIA [15] based on the TCGA database. A strong correlation was found between the mRNA levels of ROCK1 (Figure $5 \mathrm{~A})$ and ANLN $(R=0.25$, $p<0.0001$ ), as well as between ROCK2 (Figure 5 B) and ANLN $(R=0.21, p<0.0001)$. To further verify these findings, we examined activated RhoA in ANLN-overexpression Hela cells, and a significant increase in active RhoA level was observed. Furthermore, the protein levels of RhoA effectors ROCK1 and ROCK2 were found to be upregulated in ANLN-overexpressed HeLa cells (Figures 5 C, D).

To determine whether RhoA activation is involved in ANLN-induced promotion of migration and invasion in CC cells, we used Y-27632 as an inhibitor to impair the activation of RhoA in ANLNoverexpression HeLa cells. After the treatment of ANLN-overexpression cells with Y-27632 at $15 \mu \mathrm{mol}$ for $72 \mathrm{~h}$ (Figure $5 \mathrm{E}$ ), activated RhoA, ROCK1 and ROCK2 expression levels were significantly downregulated compared with the control groups (Figure $5 \mathrm{~F}, p \leq 0.05$ ). The migration and invasion of HeLa cells significantly decreased compared with those of the control groups (Figures $5 \mathrm{G}-\mathrm{I}, p \leq 0.001$ ).

\section{Discussion}

A large number of mRNAs and non-coding RNAs have been reported to play important roles in the development and progression of CC [16]. However, the molecular mechanisms underlying regulation of CC progression require further exploration and evaluation. In this study, we found that ANLN acts as a tumor promotor in CC. ANLN expression was higher in CC than in normal tissues, and high ANLN expression promoted cell proliferation, migration, and invasion in CC (Figure 2). Knockdown of ANLN disrupted the integrity of stress fibers and the RhoA/ROCK signaling pathway (Figures 3 and 4). Our results indicate that ANLN may promote the migration and invasion of CC via the RhoA/ROCK signaling pathway. 

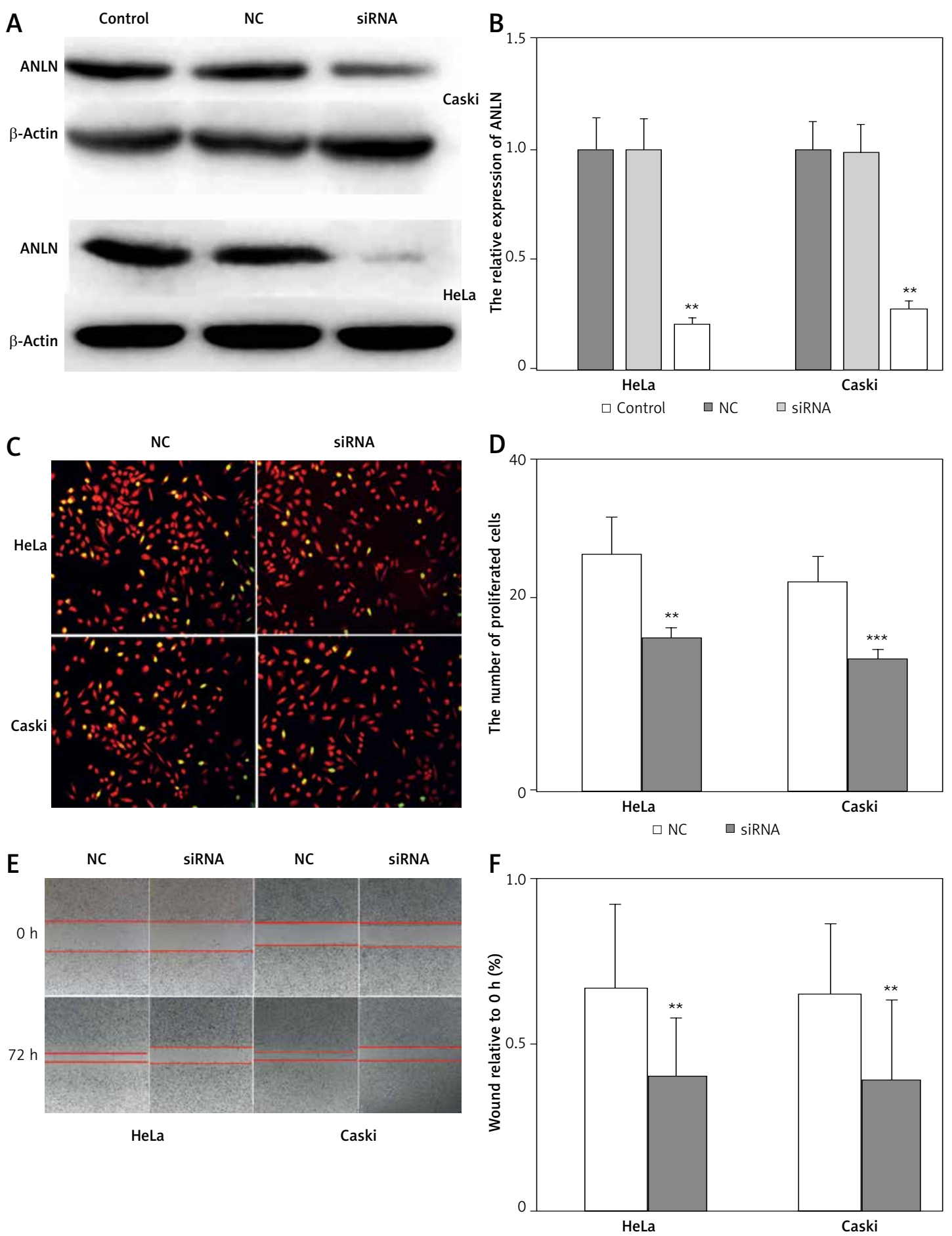

Figure 3. ANLN regulated the proliferation, migration and invasion of human cervical cells. A, B - Western blot analyses of knockdown efficiency in CC cells. $\beta$-Actin was used as the loading control. C, D - Effect of ANLN silencing on the growth of CC cells in vitro. Bar: $100 \mu \mathrm{m} . \mathrm{E}, \mathrm{F}-$ Wound closure was delayed in siRNA-transduced cells after 72 h. Bar: $200 \mu \mathrm{m}$ 
G

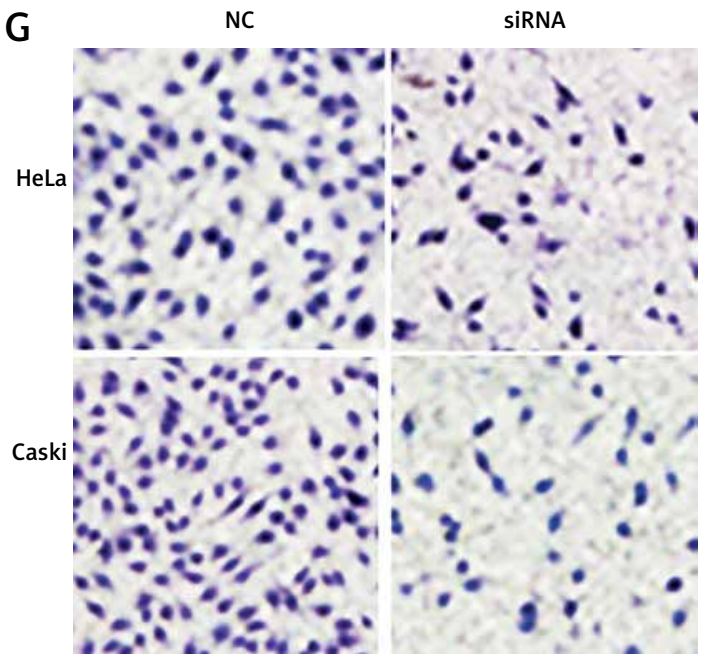

I

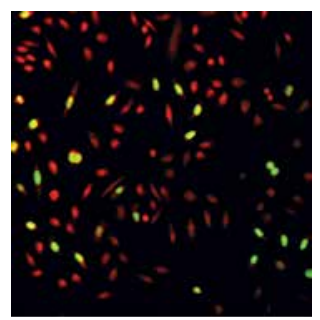

ANLN

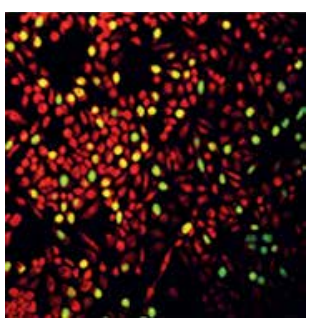

J

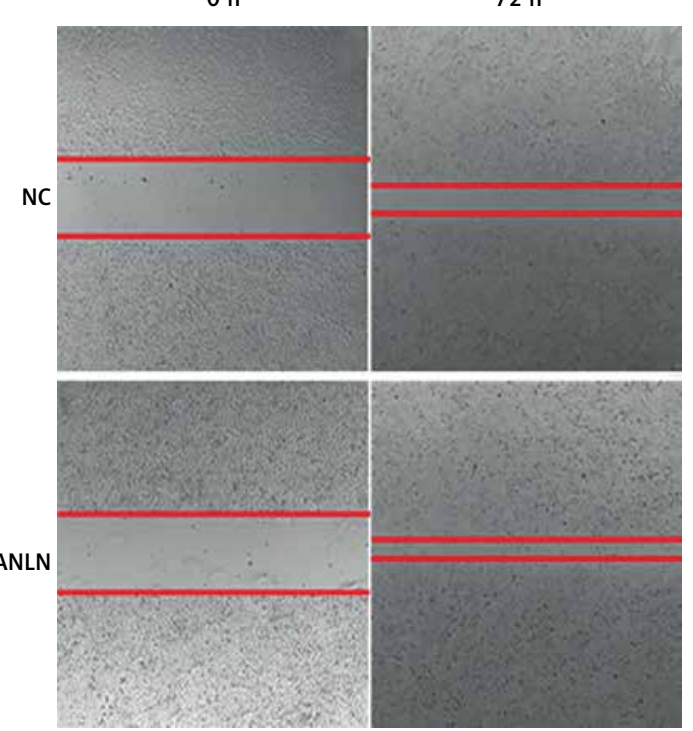

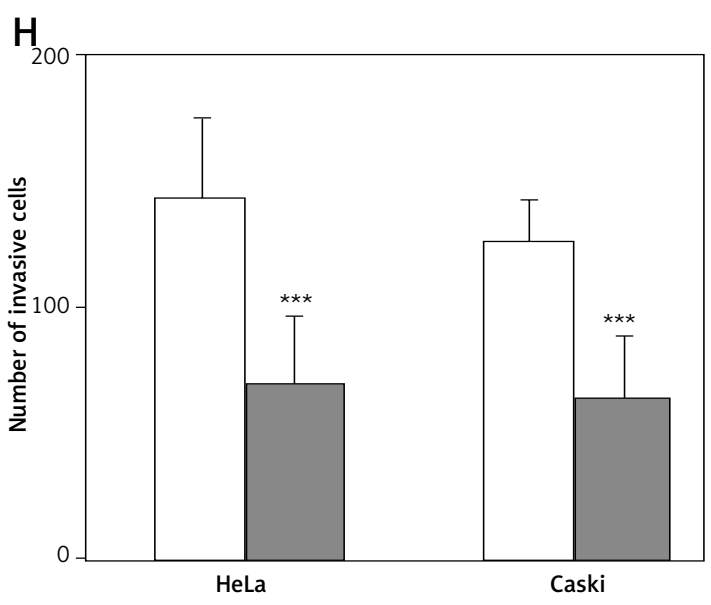
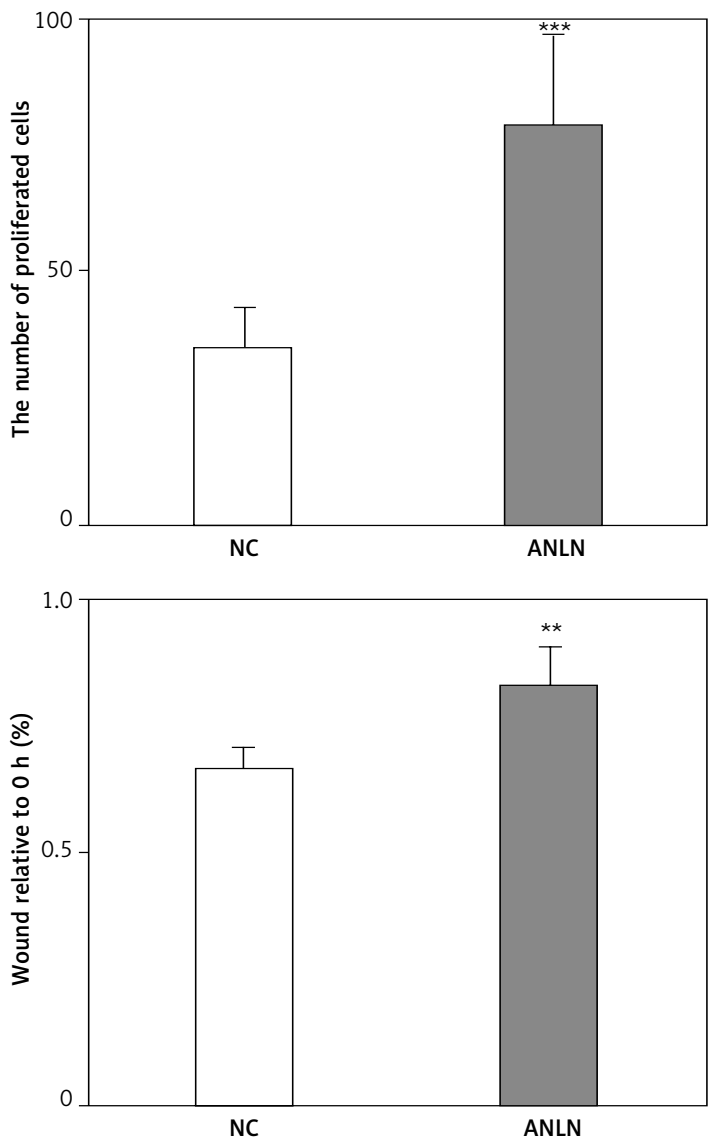

Figure 3. Cont. G, $\mathbf{H}$ - Invasion in CC cell lines decreased after transfection in a transwell assay (magnification, 200*). The number of invading cells was calculated and is depicted in the bar chart. ${ }^{*} P<0.05,{ }^{* *} p<0.01$, and ${ }^{* *} p<0.001$. I - Effect of ANLN overexpression on the growth of CC cells in vitro. Bar: $100 \mu \mathrm{m}$. J - Wound closure was delayed in ANLN-overexpressed cells after $72 \mathrm{~h}$. Bar: $200 \mu \mathrm{m}$. K - Invasion in CC cell lines increased after transfection in a Transwell assay (magnification, 200x). The number of invading cells was calculated and is depicted in the bar chart. ${ }^{*} P<0.05,{ }^{* *} p<0.01$, and ${ }^{* * *} p<0.001$ 
K

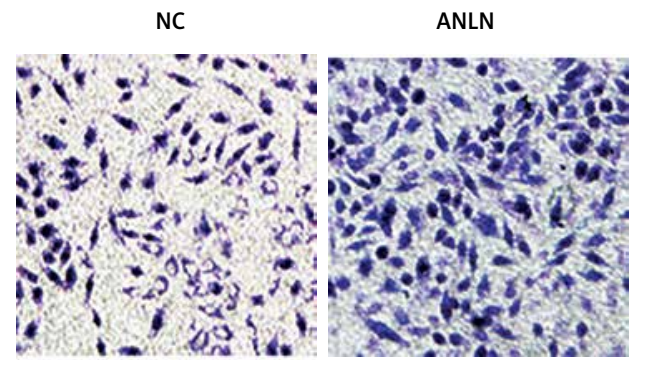

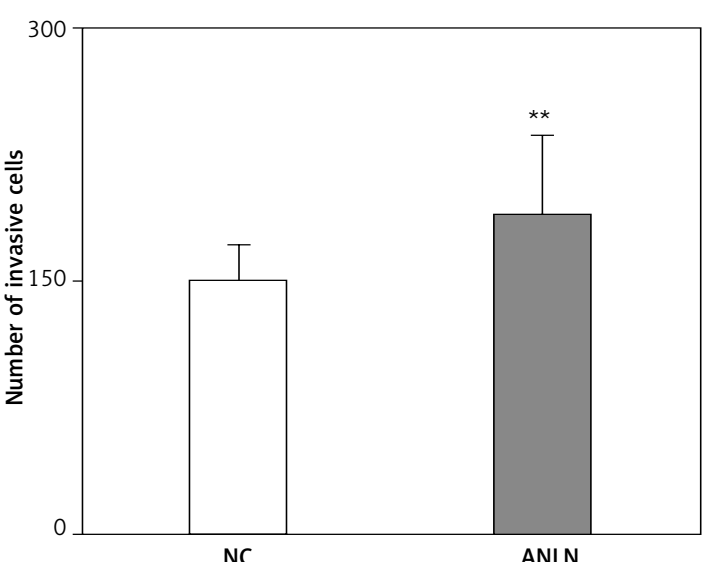

NC

ANLN

Figure 3. Cont. $\mathbf{K}$ - Invasion in CC cell lines increased after transfection in a Transwell assay (magnification, 200×). The number of invading cells was calculated and is depicted in the bar chart. ${ }^{*} p<0.05,{ }^{* *} p<0.01$, and ${ }^{* *} p<0.001$

NC

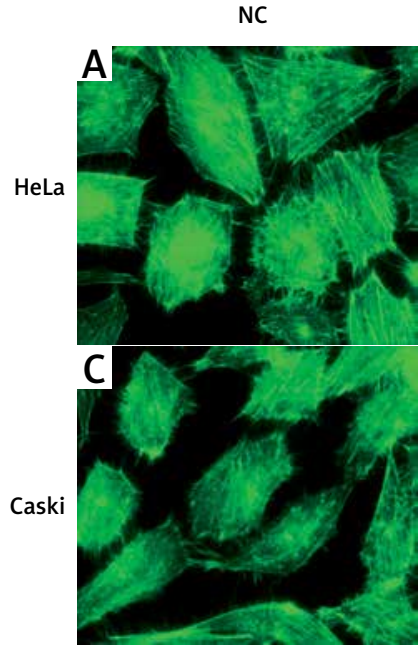

Si-ANLN

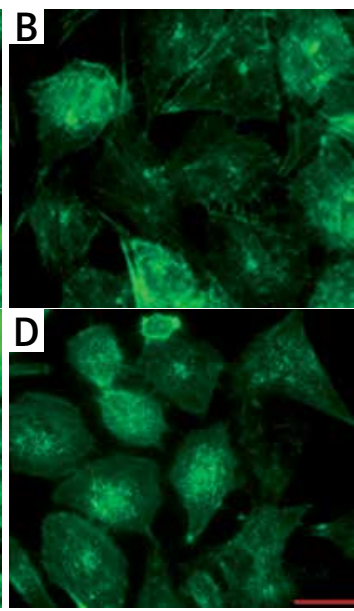

Figure 4. ANLN knockdown disrupts actin stress fiber integrity. Confocal laser scanning was used to detect the cytoskeleton morphology of CC cells transfected with siRNA or NC. Bar $=20 \mu \mathrm{m}$

A previous study involving the bioinformatic analysis of 49 CC tissues and 20 normal cervical tissues suggested that high levels of ANLN expression may represent a potential CC biomarker [14]. However, a larger number of samples is required to determine the role of ANLN in CC. In the present work, substantial samples, including 394 CC tissues and 64 normal cervical tissues, from several public databases, such as GSE7410 (Figure 1 A), GSE63514 (Figure 1 B), GSE67522 (Figure $1 \mathrm{C}$ ), TCGA, and GTEx (Genotype-Tissue Expression), were used for ANLN expression analysis. The results consistently showed that ANLN expression levels in CC are higher than those in normal tissues. 29 CC tissues and 26 normal tissues were collected from our hospital, and real-time PCR of these samples similarly showed that ANLN transcripts are more abundant in CC tissues than in normal tissues (Figure $1 \mathrm{D})$. Our findings demonstrate that ANLN could be used as a CC biomarker. We also found

that ANLN exerts tumorigenicity by promoting cell proliferation, migration, and invasion in CC. Thus, therapeutic interventions targeting ANLN may be an effective means to repress CC progression.

Research has gradually revealed a number of signaling pathways involved in the occurrence and development of CC [17-21]. The Wnt/ $\beta$-catenin signaling pathway, which is associated with cell proliferation and metastasis, is involved in cervical carcinogenesis [18, 22]. Many studies have suggested that the PI3K/Akt signaling pathway is implicated in CC progression [21, 23]. Understanding these pathways will provide new therapeutic targets to prevent CC metastasis and growth. Hence, exploration of the signaling pathways involved in CC is necessary to discover promising targets for the diagnosis and treatment of patients with this disease. In the current study, ANLN was found to maintain the integrity of stress fibers and act as an upstream regulator of the RhoA/ROCK signaling pathway in CC. The findings showed a new function of the ANLN-induced RhoA-dependent signaling pathway in CC and provide new therapeutic targets and predictive biomarkers for the disease.

In conclusion, our work identified the role and mechanisms of ANLN in CC. At the cell level, the study showed that ANLN promoted cell invasion and migration via maintaining stress fiber formation in CC cells. Furthermore, at the molecular level, our results showed that ANLN promoted cell invasion and migration through the RhoA-ROCK signaling pathway in CC. Therefore, ANLN might be a novel gene for human CC therapy. This work includes some limitations requiring further investigation. For example, in vivo experiments should be performed to validate the function of ANLN in CC. Multi-center studies with a large sample size are also needed to determine the potential of ANLN as a therapeutic target or biological marker in CC progression. 
A

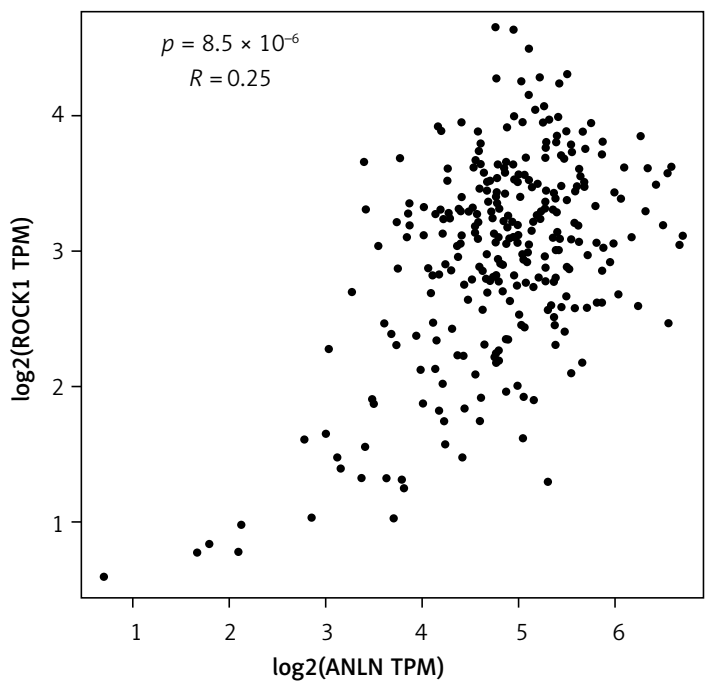

C

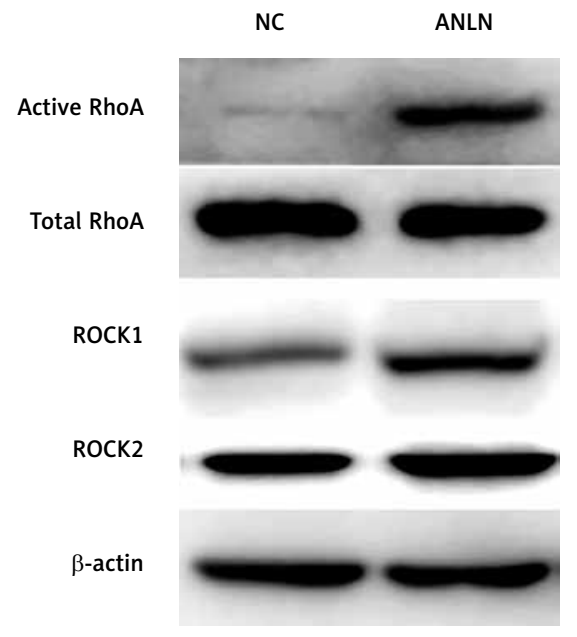

$E$

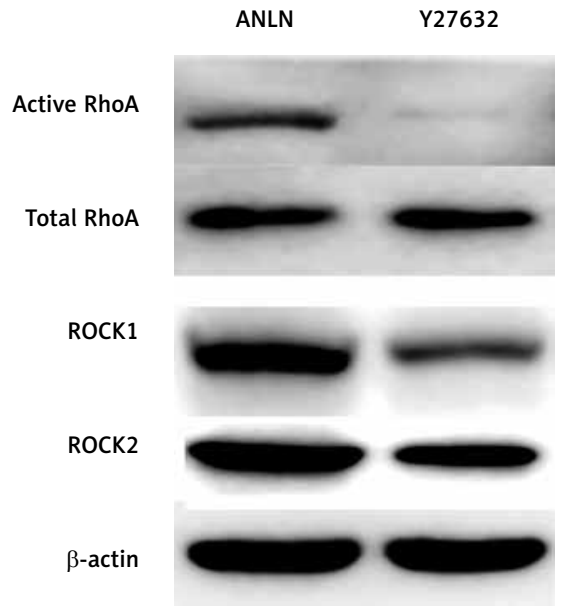

B

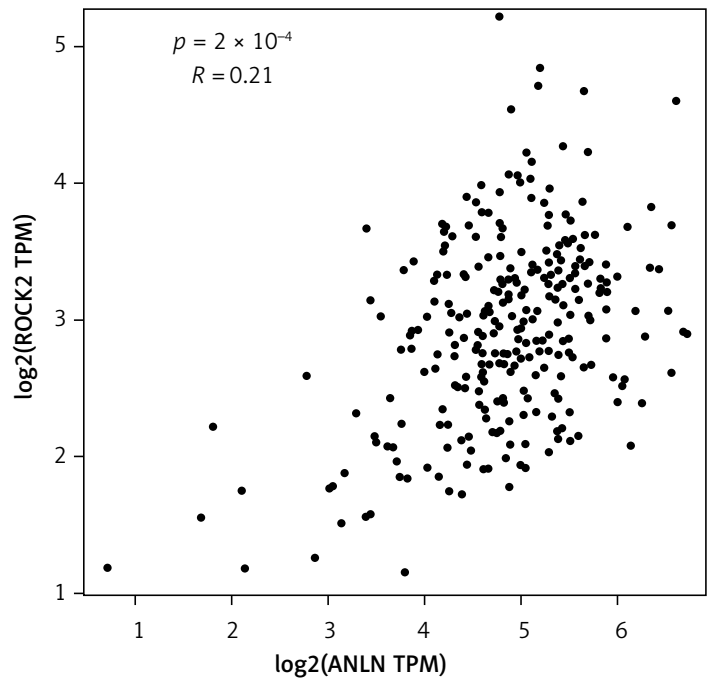

D

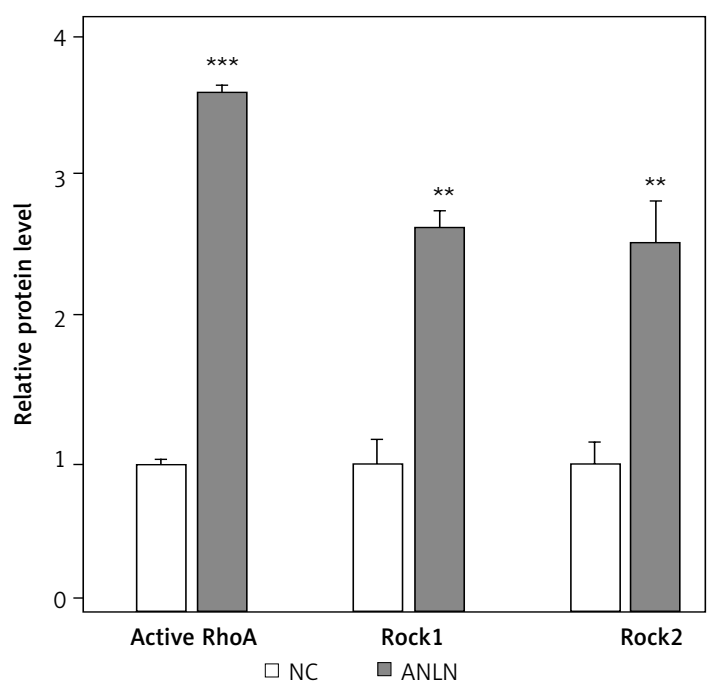

$\mathrm{F}$

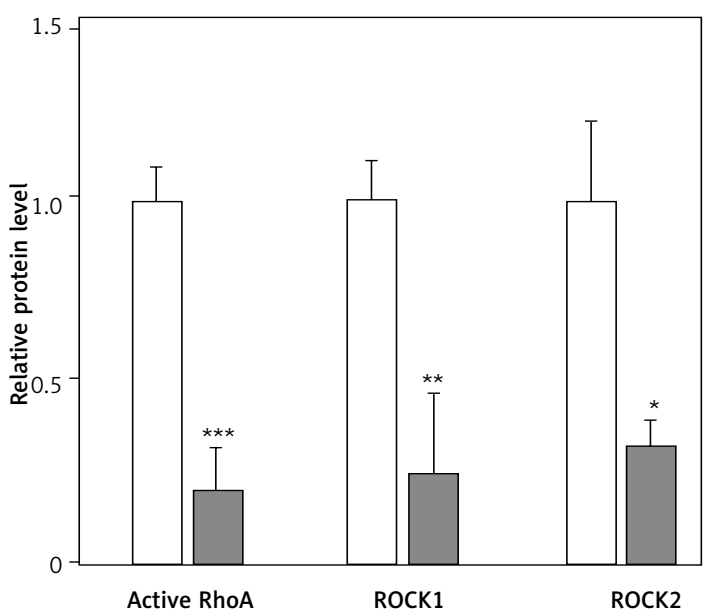

Figure 5. A, B - Scatter plots showed the association between ANLN expression and ROCK1 or ROCK2 expression using the online web portal GEPIA based on the TCGA data repository. C, D - Expression levels of active RhoA, ROCK1, and ROCK2 were detected by Western blot in ANLN-overexpression HeLa cells. E, F - Expression levels of active RhoA, ROCK1, and ROCK2 were detected by Western blot in ANLN-overexpression HeLa cells treated with Y27632 


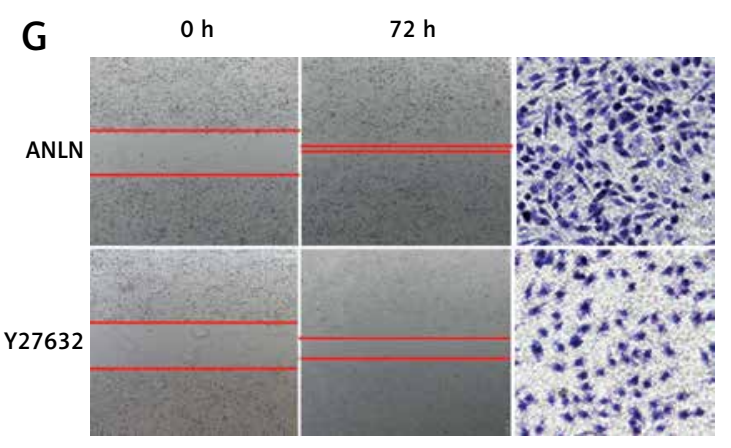

I

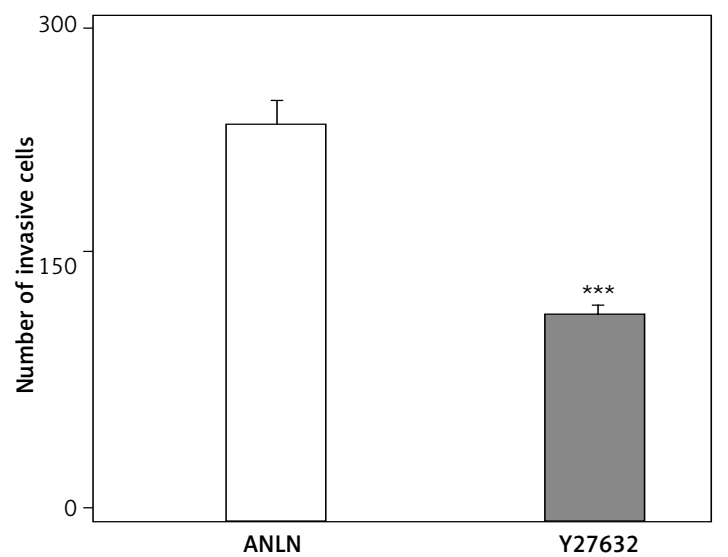

\section{Acknowledgments}

Lin Zhu and Ping Zhang contributed equally to this work.

The present study was supported by the Shandong Provincial Science and Technology Key Program (2019GSF108119), and the Nature Science Foundation of China (81801126).

\section{Conflict of interest}

The authors declare no conflict of interest.

\section{References}

1. Lewitowicz P, Nasierowska-Guttmejer, et al. HPV genotyping and p16/Ki-67 test significantly improve detection rate of high-grade cervical squamous intraepithelial lesion. Arch Med Sci 2020; 16: 87-93.

2. Arbyn M, Weiderpass E, Bruni L, et al. Estimates of incidence and mortality of cervical cancer in 2018: a worldwide analysis. Lancet Glob Health 2020; 8: e191-203.

3. Cohen PA, Jhingran A, Oaknin A, Denny L. Cervical cancer. Lancet 2019; 393: 169-82.

4. Serkies K, Jassem J. Systemic therapy for cervical carcinoma - current status. Chin J Cancer Res 2018; 30: 209-21.

5. Kedrin D, van Rheenen J, Hernandez L, Condeelis J, Segall JE. Cell motility and cytoskeletal regulation in invasion and metastasis. J Mammary Gland Biol Neoplasia 2007; 12: 143-52.

6. Gbadegesin RA, Hall G, Adeyemo A, et al. Mutations in the gene that encodes the F-actin binding protein anillin cause FSGS. J Am Soc Nephrol 2014; 25: 1991-2002.

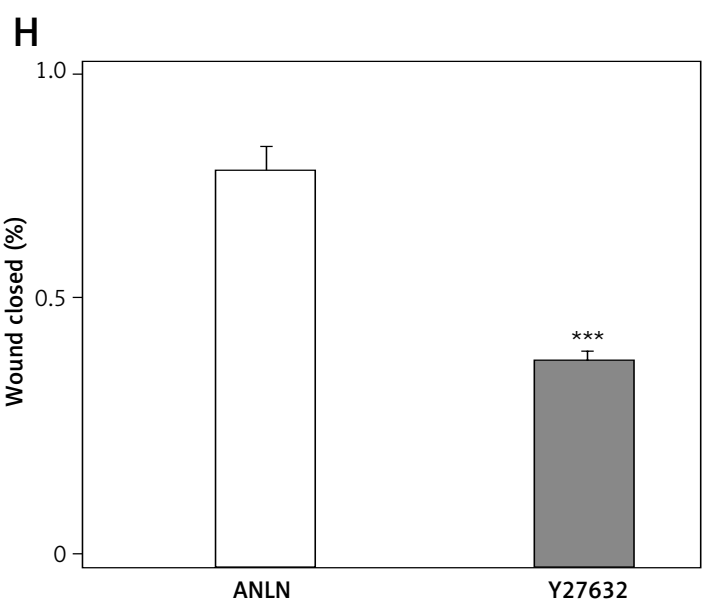

Figure 5. Cont. G-I - Y27632 could partially restore the increasing ability in migration and invasion caused by ANLN overexpression

7. Tuan NM, Lee $\mathrm{CH}$. Role of anillin in tumour: from a prognostic biomarker to a novel target. Cancers 2020; 12: 1600.

8. Pandi NS, Manimuthu M, Harunipriya P, Murugesan M, Asha GV, Rajendran S. In silico analysis of expression pattern of a Wnt/beta-catenin responsive gene ANLN in gastric cancer. Gene 2014; 545: 23-9.

9. Zeng S, Yu X, Ma C, et al. Transcriptome sequencing identifies ANLN as a promising prognostic biomarker in bladder urothelial carcinoma. Sci Rep 2017; 7: 3151.

10. Suzuki C, Daigo Y, Ishikawa N, et al. ANLN plays a critical role in human lung carcinogenesis through the activation of RHOA and by involvement in the phosphoinositide 3-kinase/AKT pathway. Cancer Res 2005; 65 : 11314-25.

11. Magnusson K, Gremel G, Ryden L, et al. ANLN is a prognostic biomarker independent of Ki-67 and essential for cell cycle progression in primary breast cancer. BMC Cancer 2016; 16: 904.

12. Wang G, Shen W, Cui L, Chen W, Hu X, Fu J. Overexpression of Anillin (ANLN) is correlated with colorectal cancer progression and poor prognosis. Cancer Biomark 2016; 16: 459-65.

13. Wang A, Dai H, Gong Y, et al. ANLN-induced EZH2 upregulation promotes pancreatic cancer progression by mediating miR-218-5p/LASP1 signaling axis. J Exp Clin Cancer Res 2019; 38: 347.

14. Xia L, Su X, Shen J, et al. ANLN functions as a key candidate gene in cervical cancer as determined by integrated bioinformatic analysis. Cancer Manag Res 2018; 10: 663-70.

15. Tang Z, Li C, Kang B, Gao G, Li C, Zhang Z. GEPIA: a web server for cancer and normal gene expression profiling and interactive analyses. Nucleic Acids Res 2017; 45: W98-102. 
16. Jiang L, Hong L, Yang W, Zhao Y, Tan A, Li Y. Co-expression network analysis of the IncRNAs and mRNAs associated with cervical cancer progression. Arch Med Sci 2019; 15: 754-64.

17. Maehama T, NIshio M, Oatni J, Mak TW, Suzuki A. The role of Hippo-YAP signaling in squamous cell carcinomas. Cancer Sci 2021; 112: 51-60.

18. Wang B, Li X, Liu L, Wang M. $\beta$-Catenin: oncogenic role and therapeutic target in cervical cancer. Biol Res 2020; 53: 33.

19. McMellen A, Woodruff ER, Corr BR, Bitler BG, Moroney MR. Wnt signaling in gynecologic malignancies. Int J Mol Sci 2020; 21: 4272.

20. Rodrigues C, Joy LR, Sachithanandan SP, Krishna S. Notch signalling in cervical cancer. Exp Cell Res 2019; 385: 111682 .

21. Bossler F, Hoppe-Seyler K, Hoppe-Seyler F. PI3K/AKT/ mTOR signaling regulates the virus/host cell crosstalk in HPV-positive cervical cancer cells. Int J Mol Sci 2019; 20: 2188.

22. Jung Y, Park J. Wnt signaling in cancer: therapeutic targeting of Wnt signaling beyond $\beta$-catenin and the destruction complex. Exp Mol Med 2020; 52: 183-91.

23. Bahrami A, Hasanzadeh M, Hassanian SM, et al. The potential value of the PI3K/Akt/mTOR signaling pathway for assessing prognosis in cervical cancer and as a target for therapy. J Cell Biochem 2017; 118: 4163-9. 